\title{
Conventions de qualité du travail et chômage de longue durée
}

\author{
Guillemette de LARQUIER ${ }^{1}$ \\ Marie SALOGNON²
}

Cette contribution ${ }^{3}$ s'inscrit dans la continuité de l'ouvrage Des marchés du travail équitables ? [Bessy et alii, 2001] qui conçoit une pluralité des inégalités légitimes en lien avec les différentes conventions possibles du bon appariement. Chaque économie crée alors ses exclus en cohérence avec la convention de qualité du travail dominante. Ici, nous traiterons d'un type précis d'exclusion : le chômage de longue durée.

Nous aurons une hypothèse de départ (l'exclusion prend sa source dans les modèles d'évaluation du travail) et une méthode (la formalisation par les modèles de durée). Nous pensons les modèles de durée assez souples d'un point de vue théorique pour se plier à l'hypothèse, elle beaucoup plus forte, que nous leur imposons. Il s'agit de prendre le contre-pied des études courantes qui imputent les dysfonctionnements du marché du travail à l'opportunisme ou l'incompétence des chômeurs. Pourquoi ne pas plutôt questionner les jugements des recruteurs, leur diffi-

\footnotetext{
1 FORUM - CNRS, Université Paris X - larquier@u-paris10.fr

2 FORUM - CNRS, Université Paris X - marie.salognon@u-paris10.fr

3 Nous tenons à remercier François Eymard-Duvernay pour sa relecture attentive et impliquée.
} 
culté à évaluer un appariement qui les amène à faire des choix sur la base de tel ou tel critère qui devient décisif, alors qu'il pourrait être remis en question? Cette posture est également celle de Marchal et Torny [2003], interrogeant la capacité des recruteurs à rédiger des annonces d'offre d'emploi : les auteurs montrent en effet sur la période 1960-2000 que la hausse des exigences formulées, la sophistication du langage employé et le manque de clarté des repères à disposition des candidats sont contre-productifs, nuisant à l'efficacité des mises en relations sur le marché.

De la sorte, nos conclusions sur le chômage de longue durée se dissocieront de celles plus connues en termes de trappe à chômage ou à inactivité. En fait, en retournant l'effet causal, on reprend un concept esquissé par Benarrosh [2000], celui de « trappe à inemployabilité », " on envisage au contraire ici que ce sont les critères minimum d'employabilité appliqués dans le tri initial des chômeurs, qui fermeraient le marché du travail aux moins lotis d'entre eux » (p. 9). À chaque modèle d'évaluation du travail correspond un concept d'employabilité, ainsi que son contingent de laissés-pour-compte au fond de la trappe, sans aucun calcul stratégique de leur part. On peut ainsi interpréter le résultat de Simonin [2000, p. 11] $]^{4}$, selon lequel près de $40 \%$ des individus n'ont eu aucun entretien pendant leur durée de chômage, de deux façons : i) leur intensité de recherche d'emploi est

\footnotetext{
${ }^{4}$ À partir de l'enquête TDE-MLT de la DARES.
} 
(maintenue) trop faible ; ii) certains profils de chômeurs ne passent jamais le cap du tri de CV et sont systématiquement écartés d'une chance d'être évalués en situation d'interaction. On ne nie pas naïvement l'existence d'arbitrage de la part des chômeurs en faveur du loisir. Nous n'accusons pas les recruteurs de discriminer volontairement une population type. Nous proposons d'explorer la piste selon laquelle, chaque convention de qualité du travail crée son « bon » profil de travailleur et, en négatif, ses inemployables pour qui les probabilités d'accès à l'emploi s'amenuisent. Seul un jugement équilibré, combinant l'ensemble des modèles d'évaluation, doit assurer une égalité des chances pour tous.

Dans une première partie, nous revenons sur le concept de convention de qualité du travail et reprenons le paramétrage d'Eymard-Duvernay [2001]. Dans la deuxième partie, ces conventions et leur paramétrage sont traduits dans le langage des modèles de durée, afin de mettre en exergue les mécanismes sous-jacents aux quatre conventions que nous aurons distinguées. La comparaison de ces mécanismes d'exclusion est réalisée dans la troisième partie. En conclusion, nous évoquerons les conventions également présentes dans les évaluations des acteurs des politiques de l'emploi en France et dans le monde anglo-saxon. 


\section{Évaluation de la qualité du travail par les conventions}

Le recrutement est une épreuve de sélection, épreuve décisive pour les chômeurs quant à leur retour à l'emploi ou leur enfoncement dans un processus d'exclusion. C'est pourquoi nous commençons par nous doter d'une mesure du degré d'équité des conventions de qualité du travail qui viennent régler ces épreuves.

Les conventions de qualité du travail : des comportements aux modèles d'évaluation

Généralement, on s'appuie sur le concept de convention pour analyser la nature des mécanismes qui permettent d'assurer la coordination entre les individus sur une base arbitraire et mal définie. Suivre une convention, c'est sélectionner une possibilité parmi des choix alternatifs en se satisfaisant d'une préférence pour la conformité, que ce choix soit optimal ou non. À ce premier niveau, les conventions sont des repères pour la coordination des comportements interindividuels (ce que Favereau [1986] appelle convention $_{2}$ ). Elles sont plurielles et arbitraires dans le sens où elles permettent toutes de se coordonner : le succès de la coordination ne dépend pas du choix d'une convention ou d'une autre. En revanche, le choix de la convention suivie dépend en général du contexte, de l'environnement au sein duquel s'opère la coordination, des objets qui appartiennent à cet environne- 
ment : les conventions ${ }_{2}$ font office de « règles ajustées aux situations $"$.

Or les conventions, au sens de l’Économie des Conventions, ne sont pas seulement des règles de comportement ou des repères pour la coordination. À un second niveau, elles sont aussi des modèles d'évaluation qui permettent d'interpréter toutes les règles, toutes les conventions 2 . Ainsi, les conventions 2 , en tant que moyens arbitraires de coordination, peuvent être jugées par les modèles d'évaluation ou, toujours selon Favereau [1986], conventions $_{1}$, ces dernières introduisant une dimension normative à l'analyse. La convention ${ }_{1}$ est alors un moyen de coordination des représentations et des jugements portés sur les comportements. Suivre une convention, c'est suivre une règle au premier niveau et en chercher le sens au second niveau. Le choix parmi plusieurs conventions ${ }_{1}$ n'est lui pas arbitraire, il désigne un monde commun soutenu par des valeurs et impliquant une conception de la justice.

Sur le marché du travail, recruter un candidat correspond à un choix à deux niveaux : choix d'un critère de sélection des candidats parmi d'autres au premier niveau, qui implique un jugement de valeur sur ce qu'est un «bon candidat » au second niveau. Par convention de qualité du travail, on entend ainsi une façon parmi plusieurs possibles d'évaluer et de qualifier le travailleur, autrement dit d'évaluer sa productivité. Si au premier niveau on peut évaluer indifféremment cette productivité de plusieurs manières, il n'est pas équivalent de mesurer la productivité du travail par le 
diplôme du travailleur, par ses résultats à des tests psychotechniques ou par un entretien d'évaluation. Ces outils de mesure relèvent effectivement de conventions de qualité différentes impliquant différentes conceptions collectives de ce qu'est un «bon travail ». Les deux niveaux d'analyse sont donc imbriqués : une convention $_{1}$ de qualité du travail est une convention constitutive qui coordonne les représentations sur ce qu'est un bon travail, d'où découle l'utilisation de certains critères pour sélectionner les «bons » travailleurs. Certaines conventions de qualité excluront plus d'individus que d'autres, induisant un problème d'inefficacité collective en termes de chômage, mais aussi un problème d'équité. Pour analyser ces deux dimensions, nous reprenons le paramétrage proposée par Eymard-Duvernay [2001], qui permet de caractériser une convention de qualité du travail selon :

- un paramètre de valeur : il décrit quel principe est mobilisé pour qualifier le travail, i.e. quel système de valeurs est au fondement de la hiérarchie des qualifications. Cette dernière peut être construite par exemple sur la base du savoir (sanctionné par le diplôme), du savoir-faire (qui renvoie aux aptitudes) ou de l'expérience.

- un paramètre d'inégalités : ce paramètre représente la plus ou moins forte inégalité de la hiérarchie des qualifications (écart relatif entre qualifications élevées et basses).

- un paramètre de renouvellement des épreuves : la qualification est attribuée une fois pour toutes ou remise en jeu pério- 
diquement.

La combinaison des deux derniers paramètres détermine le degré plus ou moins équitable de l'évaluation du travail.

Les conventions de qualité du travail mobilisées

lors du recrutement

À titre d'exemple, nous appliquons ce paramétrage aux régimes d'action des recruteurs décrits par Eymard-Duvernay et Marchal [1997]. Les auteurs distinguent deux axes de controverses entre modes d'évaluation de la compétence d'une personne. Le premier oppose les évaluations qui s'effectuent à distance des candidats (à partir de CV par exemple), à celles qui s'opèrent à proximité des candidats. Ces formes de relations ont des répercussions sur le mode d'interprétation des compétences : l'interprétation est planifiée et fondée sur des critères préétablis dans le premier cas et est négociée dans l'autre. Une deuxième dimension oppose les relations individualisées de l'évaluateur avec le candidat, aux relations qui s'insèrent dans des collectifs (institutions, réseaux, etc.). Son action vise soit à individualiser les compétences soit à considérer leur nature collective (interprétation sociologique). Le croisement de ces deux dimensions rend compte de quatre modèles d'évaluation de la compétence (conventions ${ }_{1}$ ) : l'institution, le marché, le réseau et l'interaction (cf. tableau 1). 


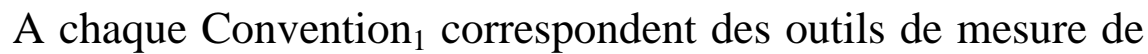
la compétence (conventions ${ }_{2}$ ). Dans le cadre d'une institution, le jugement est référé à des règles et à des catégories générales d'emploi et de candidats qui régulent les marchés internes aussi bien d'entreprises que de professions, valorisant les compétences professionnelles. Sur le marché, le recruteur sélectionne le meilleur candidat par un choix de rentabilité économique, fondé sur les aptitudes des candidats, autrement dit leur productivité ou leur potentiel. L'appui sur un réseau de relations permet au recruteur de se fier à des réputations transmises de proche en proche et d'apprécier l'expérience d'un candidat en situation. Les compétences des acteurs sont distribuées dans leur réseau de relations. En situation d'interaction avec le candidat, l'évaluateur cherche à instaurer une relation de confiance avec lui en se fondant sur son intuition. Les compétences des candidats sont considérées comme émergentes, dans la mesure où elles résultent du déroulement de l'entretien.

TABLEAU 1 : LES CONVENTIONS DE QUALITÉ DU TRAVAIL

\begin{tabular}{|c|c|c|}
\cline { 2 - 3 } \multicolumn{1}{c|}{} & $\begin{array}{c}\text { Interprétation } \\
\text { planifiée }\end{array}$ & $\begin{array}{c}\text { Interprétation } \\
\text { négociée }\end{array}$ \\
\hline $\begin{array}{c}\text { Interprétation } \\
\text { individualiste }\end{array}$ & Marché & Interaction \\
\hline $\begin{array}{c}\text { Interprétation } \\
\text { sociologique }\end{array}$ & Institution & Réseau \\
\hline
\end{tabular}

in F. Eymard-Duvernay (ed.), Valeurs, coordination et rationalité - développements, La Découverte, coll. Recherche, avril, pp. 111-127 
Nous pouvons appliquer le paramétrage à ces différentes conventions de qualité. Pour chacune d'elles, si le paramètre de valeur conditionne celui de renouvellement des épreuves, il ne semble cependant pas pertinent de les classer selon le paramètre d'inégalités. En effet, au sein de chaque convention, l’inégalité de la hiérarchisation peut être plus ou moins forte. Il n’y a pas un régime d'action plus inégal qu'un autre dans l'absolu. C'est pourquoi sur les trois paramètres, on ne spécifie que les paramètres de valeur et de renouvellement des épreuves, en précisant les outils sur lesquels s'appuie l'évaluation.

- Dans la convention de qualité «marché », les outils de la coordination sont les tests psychotechniques. Le paramètre de valeur correspond aux aptitudes individuelles (motivation, autonomie, personnalité...) et l'épreuve est non renouvelée car les aptitudes sont supposées ancrées dans la personne, l'évaluation est planifiée.

- La convention «institution» utilise les CV, concours, dossiers comme objets de coordination. Les candidats sont valorisés par leurs compétences professionnelles (diplôme, grade, grille de qualifications selon la convention collective) et l'épreuve d'évaluation est peu renouvelée, car les compétences sont supposées planifiées par l’institution.

- La convention « interaction » s'appuie sur l'entretien en face à face comme outil de coordination. Ce sont les compétences émergentes des candidats (produit de l'ajustement inte- 
rindividuel, de l'intuition du recruteur) qui sont valorisées et l'épreuve est renouvelée puisque l'évaluation de la compétence est supposée émerger dans les interactions.

- Dans la convention « réseau », les acteurs s'appuient sur la recommandation par des tiers pour se coordonner. Ils évaluent les travailleurs en fonction de leurs compétences distribuées (réputation, expérience) et l'épreuve peut être renouvelée, puisque l'évaluation de la compétence émerge dans le réseau.

L'application aux Façons de recruter permet d'identifier les travailleurs exclus de l'emploi selon telle ou telle convention. Toutefois, elle omet l'analyse explicite de la durée de chômage, indispensable pour caractériser les processus d'exclusion. Cela nous prive également d'un critère d'efficacité simple : le taux de chômage de longue durée. Afin d’y remédier, nous avons recours à la formalisation avec les modèles de durée.

\section{Une modélisation conventionnaliste des durées de chômage}

La méthode que nous utilisons est connue : partir d'un modèle de durée générique et y introduire différentes hypothèses afférentes aux mécanismes de sortie du chômage. Néanmoins, notre étude se distingue des analyses devenues courantes séparant essentiellement les causes d'hétérogénéité ex ante et ex post. Ce 
n'est pas le constat d'un «tri » possible des chômeurs dès le départ ou au contraire fonction de la durée qui retient notre attention, nous cherchons plutôt à relier équité de la convention et chômage. Plus précisément, la « convention de qualité » est représentée par un ensemble de variables déterminant les embauches auxquelles on associe les paramètres de valeur et d'équité (renouvellement des épreuves et inégalités). La pluralité des combinaisons fera la pluralité des conventions.

Le modèle générique

On se place sur un marché du travail où le salaire $w$ est fixe (pour des considérations d'efficience dans le marché interne ou du fait d'une régulation externe de branche). En revanche, le surplus produit $\rho$ peut varier d'un appariement à l'autre. L'enjeu de l'entretien d'embauche est alors pour l'entreprise d'évaluer le surplus net de l'appariement $(\rho$-w) qui lui reviendra. Pour l'estimer, le recruteur de l'entreprise interprète les signaux ou indices disponibles à l'aide d'un critère, noté $A$. À l'aune de ce critère, le candidat à un emploi est évalué ou « qualifié » sur une échelle normée de 0 à 1 , sa note ou qualification est donnée par la fonction $q(a+k)$, avec $a$ une variable propre à l'individu $(0 \leq a \leq 1)$ et $k$ une variable locale attachée à l'appariement.

Le critère $A$ représente ainsi le paramètre de valeur, et l'opération de qualification varie avec ce dernier, selon deux dimensions : 
1. La qualification diminue-t-elle avec l'éloignement pris par rapport à l'emploi ou est-elle indépendante de la durée de chômage ?

2. La qualification est-elle universelle ou locale ?

Ces deux dimensions ont partie liée avec la question du renouvellement possible ou non de l'épreuve de qualification. Par exemple, si l'on considère que le critère perd de sa valeur avec la durée de chômage, la probabilité d'entretien avec un recruteur diminue, l'épreuve est de moins en moins souvent renouvelée ; ou encore, si le critère est universel, il peut y avoir renouvellement de l'épreuve mais non de la qualification finale (en quelque sorte le renouvellement est fictif). Comme il a été écrit plus haut, caractériser le critère de valeur d'une convention de qualité nous donne le degré de renouvellement possible ou non de l'épreuve.

Formellement, on représente ces deux dimensions à l'aide respectivement d'une fonction $m(s)$ et de la variable $k$.

Soit $s$ la durée de chômage $(s \geq 0)$. Soit $\mu$ la probabilité «théorique » de rencontrer un employeur (fonction uniquement de la situation macro-économique, par exemple, du rapport entre postes vacants et chômeurs : $0<\mu<1)$. La fonction $m(s)$ est l'impact de la durée de chômage sur la probabilité de rencontre. Un chômeur depuis s périodes a donc une probabilité d'être effectivement évalué par un recruteur égale à $\mu m(s)$ où $m(0)=1$. Deux cas sont possibles :

i) $m(s)=1 \quad \forall s$ : la qualification est considérée indépendante de l'éloignement pris par rapport à l'emploi (la probabi- 
lité de rencontre $\mu m(s)=\mu$ est constante, les recruteurs n'écartent pas a priori de l'évaluation les individus depuis longtemps hors de l'emploi) ;

ii) $d m / d s<0$ et $\lim _{s \rightarrow+\infty} m(s)=0$ : la qualification est considérée fonction de l'éloignement pris par rapport à l'emploi (la probabilité de rencontre $\mu m(s)$ décroît avec $s$, les recruteurs écartent $a$ priori de l'évaluation les individus depuis longtemps hors de l'emploi).

Par ailleurs, avec $k$ le caractère plus ou moins local du critère qui vient affecter la variable $a$, la fonction de qualification $q(a+k)$, résumant l'action du recruteur s'écrit :

$$
q(a+k)=\left\{\begin{array}{l}
0 \text { si } a+k<0 \\
a+k \quad \text { si } 0 \leq a+k \leq 1 . \\
1 \quad \text { si } a+k>1
\end{array}\right.
$$

De nouveau, on envisage deux cas :

i) soit $k=0$ : la qualification est universelle $(q(a+k)=a)$;

ii) soit $k$ suit une loi uniforme sur $[-1,+1]$ (avec $G$ sa fonction de répartition) : la qualification est locale.

\section{La typologie}

À présent, croisant les deux dimensions, on construit une typologie de conventions de qualité établissant quatre «valeurs » alternatives ( $c f$. tableau 2) : « aptitudes individuelles », « compétences professionnelles », « compétences émergentes » et « compétences distribuées dans un réseau ». À chacune correspond une 
fréquence possible du renouvellement de l'épreuve de qualification.

TABleAu 2. TyPOLOGIE DES CONVENTIONS DE QUALITÉ

\begin{tabular}{|c|c|c|}
\cline { 2 - 3 } \multicolumn{1}{c|}{} & $\begin{array}{c}\text { La qualification est uni- } \\
\text { verselle } \\
k=0\end{array}$ & $\begin{array}{c}\text { La qualification est lo- } \\
\text { cale } \\
k \in[-1,+1]\end{array}$ \\
\hline $\begin{array}{c}\text { La qualification est } \\
\text { considérée indépendante } \\
\text { de l'éloignement pris par } \\
\text { rapport à l'emploi } \\
m(s)=1, \forall s\end{array}$ & $\begin{array}{c}\text { aptitudes individuelles } \\
\text { renouvellement fictif }\end{array}$ & $\begin{array}{c}\text { compétences émergen- } \\
\text { tes }\end{array}$ \\
renouvellement existant \\
$\begin{array}{c}\text { La qualification est } \\
\text { considérée fonction de } \\
\text { l'éloignement pris par } \\
\text { rapport à l'emploi } \\
d m / d s<0\end{array}$ & $\begin{array}{c}\text { compétences profes- } \\
\text { sionnelles } \\
\text { renouvellement fictif et } \\
\text { décroissant }\end{array}$ & $\begin{array}{c}\text { compétences distri- } \\
\text { buées dans un réseau } \\
\text { renollement décrois- } \\
\text { sant }\end{array}$ \\
\hline
\end{tabular}

Nous retrouvons quatre conventions d'évaluation du travail assez proches des régimes de recrutement d'Eymard-Duvernay et Marchal [1997]. De fait, il y a pour nous parfaite équivalence entre notre opposition universelle / locale et leur opposition planifiée / négociée. En revanche, la dimension « éloignement ou non par rapport à l'emploi », qui nous permet d'analyser explicitement un phénomène de dépendance temporelle, peut paraître distincte du caractère individualiste ou sociologique de l'évaluation. Pourtant, le rapprochement des deux axes est moins audacieux si l'on songe qu'il s'agit de noter si une position encore récente dans un collectif institutionnel ou réticulaire de pro- 
duction pèse ou non dans la qualification.

À ces quatre conventions de qualité du travail, nous refusons d'associer systématiquement un paramètre d'inégalités. Même la convention « aptitudes individuelles » pourrait être extrêmement égalitaire si toutes les aptitudes étaient considérées productives ou employables. Il faut donc introduire une dimension supplémentaire : le niveau de sélection. L’aspect inégalitaire du recrutement se déduit du seuil minimal $\bar{a}(0 \leq \bar{a} \leq 1)$ en dessous duquel l'entreprise refuse d'embaucher (i.e. tous les candidats tels que $q(a+k)<\bar{a}$ sont écartés).

Avec $F$ la fonction de répartition de $a, F(\bar{a})$ est donc la proportion de la population inemployable au sens du critère $A$ si celui-ci est universel. Dans le cas où le paramètre de valeur n'est pas universel, la notion de population inemployable n'a plus de sens : individuellement tout chômeur a une probabilité non nulle d'être localement évalué employable. Néanmoins, même dans ce dernier cas, la probabilité décroît avec le niveau de sélection $\bar{a}$.

Fonctions de risque et de survie

Soit $\pi(s \mid a)$ le risque de sortie du chômage, après une durée de chômage $s$, sachant la qualification $a$ :

$$
\pi(s \mid a)=\mu m(s) \operatorname{Prob}(q(a+k) \geq \bar{a}) .
$$


Pour des raisons de cohérence ${ }^{5}$ et de continuité ${ }^{6}$, on pose $\bar{a} \neq 0$, d’où:

$$
\pi(s \mid a)=\mu m(s) \operatorname{Prob}(a+k \geq \bar{a})
$$

Du taux instantané de sortie $\pi(s \mid a)$, on déduit la fonction de survie ${ }^{7}$ $H(s \mid a)$ des individus de qualification $a$ après $s$ périodes de chômage [Kiefer, 1988, p. 652] :

$$
H(s \mid a)=\exp \left[-\int_{0}^{s} \pi(t \mid a) \mathrm{d} t\right]
$$

Pour obtenir la fonction de survie de la cohorte entière $H(s)$, il suffit d'intégrer l'expression /3/ par rapport à la variable $a$ sur l'intervalle $[0,1]$.

$$
H(s)=\int_{0}^{1} \exp \left[-\int_{0}^{s} \pi(t \mid a) \mathrm{d} t\right] \mathrm{d} a
$$

La densité des chômeurs de qualification $a$ parmi tous les chômeurs d'ancienneté $s$ est alors [Decreuse et di Paola, 2002, p. 208] :

$$
\phi(a \mid s)=\frac{H(s \mid a)}{H(s)}=\frac{\exp \left[-\int_{0}^{s} \pi(t \mid a) \mathrm{d} t\right]}{\int_{0}^{1} \exp \left[-\int_{0}^{s} \pi(t \mid \alpha) \mathrm{d} t\right] \mathrm{d} \alpha}
$$

On peut enfin écrire $p(s)$, le risque moyen de sortie du chômage des chômeurs d'ancienneté $s$, toutes qualifications confondues :

$$
p(s)=\int_{0}^{1} \phi(a \mid s) p(s \mid a) \mathrm{d} a
$$

\footnotetext{
${ }^{5}$ Dans le cas où $d m / d s<0$, il faut rejeter l'hypothèse $\bar{a}=0$. De fait, si le recruteur interprète la durée de chômage comme un indice sur la valeur du candidat, il exclut de l'épreuve de qualification certains individus parce qu'il estime a priori un niveau $q(a+k)$ trop faible. Il serait alors absurde de supposer qu'a posteriori le recruteur est prêt à accepter tous les candidats.

${ }^{6}$ Dans le cas $\bar{a}=0$, on aurait un problème de discontinuité en 0 , qui ne permettrait pas de raisonner de manière équivalente à partir de $q(a+k)$ et de $a+k$.

${ }^{7}$ Il s'agit de la fraction d'individus entrés au chômage à la même date et toujours chômeurs après une durée $s$.
} 
Les quatre modèles de durée ${ }^{8}$

Pour caractériser les processus d'exclusion, on décline pour chaque convention de qualité la fonction de risque individuel de sortie et les fonctions de survie et de risque moyen.

La convention « aptitudes individuelles »

Cette convention correspond au cas répertorié dans la littérature standard où seule agit une pure hétérogénéité ex ante. L'équation /2.b/ devient :

$$
\pi(s \mid a)= \begin{cases}\mu & \text { si } \quad a \geq \bar{a} \\ 0 & \text { sinon }\end{cases}
$$

Non seulement le risque individuel de sortie du chômage est indépendant de la durée, mais encore il ne peut prendre que deux valeurs dans l'ensemble de la population. Si un chômeur est qualifié inemployable de manière universelle $(a<\bar{a})$, sa probabilité de retourner dans l'emploi est nulle dès le départ. En revanche, une chômeuse qualifiée $a>\bar{a}$ a un risque constant $\mu$ de retrouver un emploi. Dans ces conditions, le risque moyen décroît au rythme de sortie des chômeurs suffisamment bien qualifiés.

Les calculs donnent respectivement pour la fonction de survie et celle de risque moyen :

\footnotetext{
${ }^{8}$ Le détail des calculs est disponible auprès des auteurs.
} 


$$
\begin{gathered}
H(s)=\int_{0}^{\bar{a}} \mathrm{~d} a+\int_{\bar{a}}^{1} \exp (-\mu s) \mathrm{d} a=\bar{a}+(1-\bar{a}) \exp (-\mu \mathrm{s}) \\
p(s)=\frac{(1-\bar{a}) \mu /}{1+\bar{a}(\exp (\mu s)-1)}
\end{gathered}
$$

À long terme ${ }^{9}$, la fraction inemployable reste irréductiblement au chômage : il y a bien exclusion due à une hétérogénéité ex ante universellement admise et jamais remise en question. En reprenant les termes de Benarrosh [2000], il y a « trappe à inemployabilité ».

\section{La convention « compétences professionnelles »}

Cette convention garde le caractère universel des qualifications, discriminant les chômeurs et chômeuses employables des inemployables . À cela s’ajoute l'idée que la qualification est liée à l'occupation encore récente d'un poste dans un collectif de production. La durée de chômage est donc interprétée de manière négative. Le risque individuel de sortie du chômage intègre $m(s)$ dans l'expression /7/ :

$$
\pi(s \mid a)=\left\{\begin{array}{l}
\mu m(s) \text { si } a \geq \bar{a} \\
0 \quad \text { sinon }
\end{array}\right.
$$

Cette fois-ci, le risque individuel de sortie du chômage est dépendant de la durée pour la partie employable de la population : tous les individus $a>\bar{a}$ voient leur chance de retrouver un em-

$9 \lim _{s \rightarrow+\infty} H(s)=\bar{a}$ 
ploi diminuer au même rythme. En revanche, si de manière universelle une chômeuse est qualifiée à un niveau $a<\bar{a}$, sa probabilité de retourner dans l'emploi est constante et nulle. Le risque moyen ne dépend donc que de la vitesse de sortie des employables, comme pour la convention «aptitudes individuelles ». La fonction de survie s'écrit :

$$
H(s)=\bar{a}+(1-\bar{a}) \exp \left(-\mu \int_{0}^{s} m(t) \mathrm{d} t\right)
$$

La convention « compétences professionnelles » place de la même façon les inemployables au fond d'une « trappe ».

La fonction de risque moyen prend la forme :

$$
p(s)=\frac{(1-\bar{a}) \mu m(s)}{1+\bar{a}\left(\exp \left[\mu \int_{0}^{s} m(t) \mathrm{d} t\right]-1\right)}
$$

La convention « compétences émergentes »

Le caractère totalement local de la qualification et le fait que la durée de chômage ne présage rien de l'évaluation changent plus profondément les expressions mathématiques. Et le changement n'est pas moindre pour l'interprétation puisque, dorénavant, la catégorie des travailleurs employables n'a plus de sens. L'équation /2.b/ devient :

$$
\pi(s \mid a)=\mu \frac{1+a-\bar{a}}{2}
$$

Le risque individuel de sortie du chômage est indépendant de la durée de chômage $s$. Le niveau élevé ou faible de ce risque 
dépend uniquement de la qualification qui, étant donné son évaluation locale (émergeant lors de l'entretien), a une probabilité $\frac{1+a-\bar{a}}{2}$ d'être suffisante pour mener à un recrutement ferme. Même avec $a=0$, un chômeur ne peut être irrémédiablement exclu de l'emploi : on vérifie que $\pi(s \mid 0)=\mu \frac{1-\bar{a}}{2} \geq 0$ puisque $\bar{a} \leq 1$. Inversement, une chômeuse de niveau $a$ élevé (mais strictement inférieur à 1) n’a jamais la garantie d'être embauchée à la fin d'un entretien $\left(\frac{1+a-\bar{a}}{2}<1\right.$ pour $\left.a<1\right)$. Il faut alors assimiler la variable $a$ non plus à une qualification ordonnant une bonne fois pour toutes l'ensemble des chômeurs, mais plutôt à une probabilité d'être gagnant lors d'une évaluation locale.

On exprime les deux fonctions habituelles (pour les valeurs non nulles de $s$ ) :

$$
\begin{aligned}
& H(s)=\frac{2}{\mu s}\left[1-\exp \left(\frac{-\mu s}{2}\right)\right] \exp \left(-\mu s \frac{1-\bar{a}}{2}\right) \\
& p(s)=\frac{\mu}{2}\left[1-\bar{a}+\frac{2}{\mu s}-\frac{1}{\exp \left(\frac{\mu s}{2}\right)-1}\right]
\end{aligned}
$$

Cette fois-ci, la fonction de survie de la cohorte tend vers 0 : une évaluation des chômeurs selon les « compétences émergentes » permet à très long terme la sortie vers l'emploi de tous les chômeurs.

La convention « compétences distribuées dans un réseau » 
Il s’agit enfin de mixer les résultats précédents fondés sur le caractère local de l'évaluation avec les effets négatifs d'une baisse du taux de rencontre, due à la perte d'efficacité des liens dans le réseau. La fonction de risque individuel se déduit facilement de $/ 13 /$ :

$$
\pi(s \mid a)=\mu m(s) \frac{1+a-\bar{a}}{2}
$$

De nouveau, il n’y a pas de population stigmatisée inemployable, $\pi(s \mid 0)=\mu \frac{1-\bar{a}}{2} \geq 0$. En revanche, pour tous les chômeurs, le risque de sortie vers l'emploi décroît avec la durée de chômage.

Pour la dernière fois (pour les valeurs non nulles de $s$ ) :

$$
\begin{gathered}
H(s)=\frac{2}{\mu \int_{0}^{s} m(t) \mathrm{d} t}\left[1-\exp \left(\frac{-\mu \int_{0}^{s} m(t) \mathrm{d} t}{2}\right] \exp \left(-\mu \int_{0}^{s} m(t) \mathrm{d} t \frac{1-\bar{a}}{2}\right)\right. \\
p(s)=\frac{\mu m(s)}{2}\left[1-\bar{a}+\frac{2}{\mu \int_{0}^{s} m(t) \mathrm{d} t}-\left[\exp \left(\frac{\mu \int_{0}^{s} m(t) \mathrm{d} t}{2}\right)-1\right]\right.
\end{gathered}
$$

\section{À chaque convention, son chômage de longue durée}

Dans cette dernière partie, nous comparons en termes d’équité 
et d'efficacité les quatre conventions. Pour fixer les idées, on a simulé les fonctions de risque et de survie associées dans les cas de faible et forte sélections (niveau du paramètre d’inégalités), avec $m(s)=\exp (-s / v)$, où $v$ est un paramètre strictement positif (cf. graphiques 1 à 4$)$.

GRAPHIQUE 1. LES FONCTIONS DE RISQUE SIMULÉES DES QUATRE CONVENTIONS DE QUALITÉ AVEC FAIBLE SÉLECTION :

$$
\overline{\boldsymbol{a}}<0.5(\overline{\boldsymbol{a}}=0.1 ; \mu=0.7 ; \mathrm{V}=10)
$$

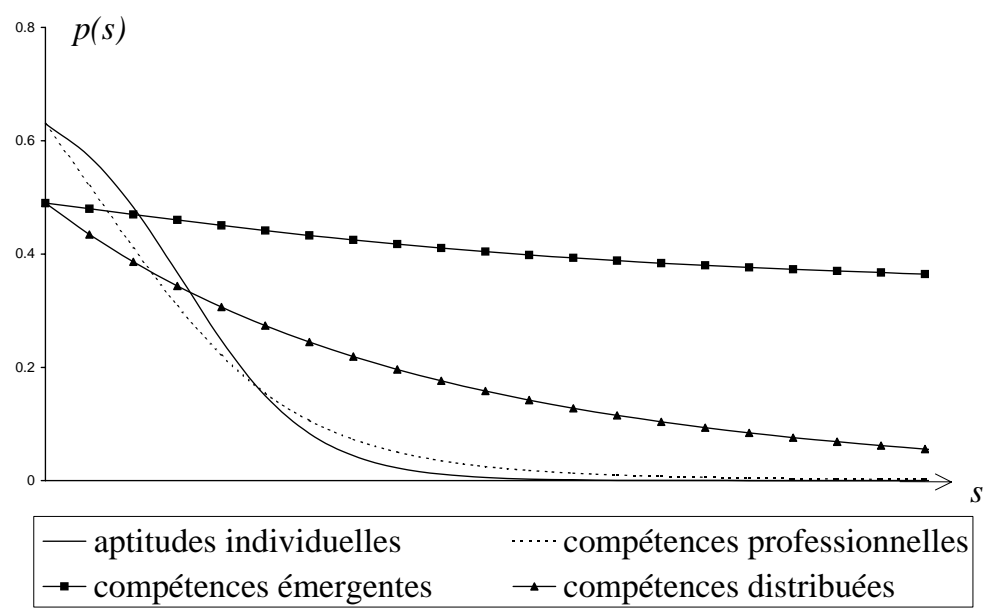

Les graphiques 1 et 2 renseignent sur l'évolution de la probabilité de sortie du chômage avec la durée de chômage selon la convention de qualité du travail adoptée lors du recrutement. On peut les interpréter en termes d’équité : à degré de sélection donné, et donc à paramètre d'inégalités $\bar{a}$ fixé, ce sont les courbes les plus plates qui traduisent une plus grande égalité d'accès à l'emploi pour l'ensemble des chômeurs, quelle que soit leur du- 
rée de chômage. Il s’agit des conventions « compétences émergentes » et "compétences distribuées ». Le renouvellement de l'épreuve atténue l'inégalité des chances de sortie entre les chômeurs, chances qui perdurent. À l'inverse, les courbes les plus pentues sont aussi les plus inégalitaires. Il s'agit des conventions « aptitudes individuelles » et «compétences professionnelles » pour lesquelles la probabilité de sortie chute rapidement. En effet, elles n'admettent pas le renouvellement de l'évaluation et induisent une sortie du chômage très rapide pour les « bons » et un chômage de longue durée pour les inemployables, qui voient finalement disparaître toute chance de sortir de leur « trappe ».

GRAPHIQUE 2. LES FONCTIONS DE RISQUE SIMULÉES DES QUATRE CONVENTIONS DE QUALITÉ AVEC FORTE SÉLECTION : $\overline{\boldsymbol{a}}>0.5(\overline{\boldsymbol{a}}=0.7 ; \mu=0.7 ; \mathrm{V}=10)$

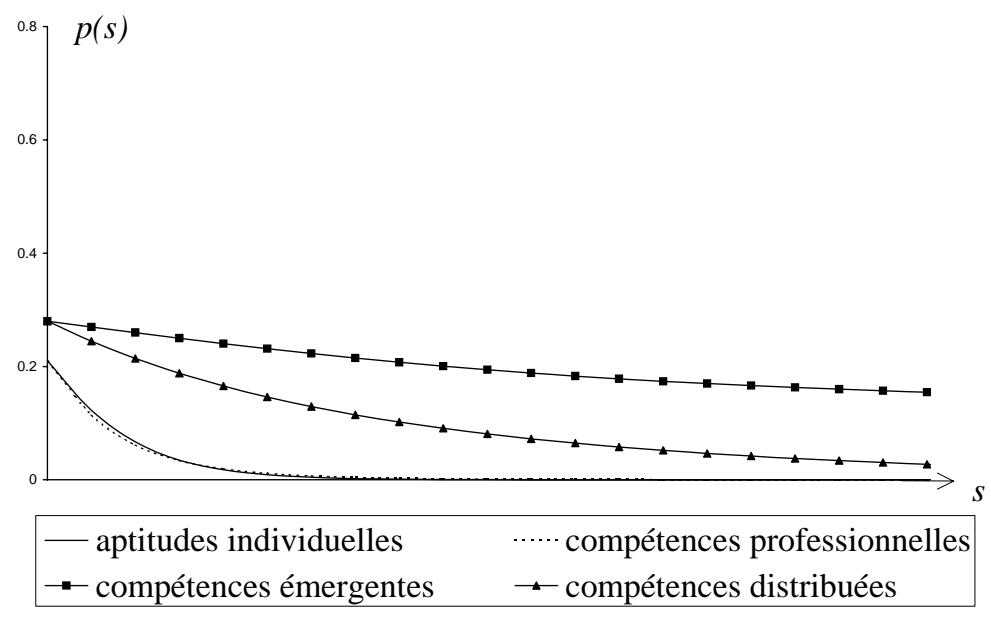

Le degré de sélection joue sur l'ordonnée à l'origine, donc sur 
les probabilités de sortie au début de la période de chômage. Quelle que soit la convention de qualité adoptée, cette probabilité chute avec le durcissement de la sélection, mais dans des proportions bien plus fortes concernant les conventions « aptitudes individuelles » et « compétences professionnelles ». Toutefois, la forme des quatre fonctions varie relativement peu avec le degré de sélection, confirmant notre interprétation précédente des courbes en termes d'égalité d'accès à l'emploi.

GRAPHIQUE 3. LES FONCTIONS DE SURVIE SIMULÉES

DES QUATRE CONVENTIONS DE QUALITÉ AVEC FAIBLE SÉLECTION :

$$
\overline{\boldsymbol{a}}<0.5(\overline{\boldsymbol{a}}=0.1 ; \mu=0.7 ; \mathrm{V}=10)
$$

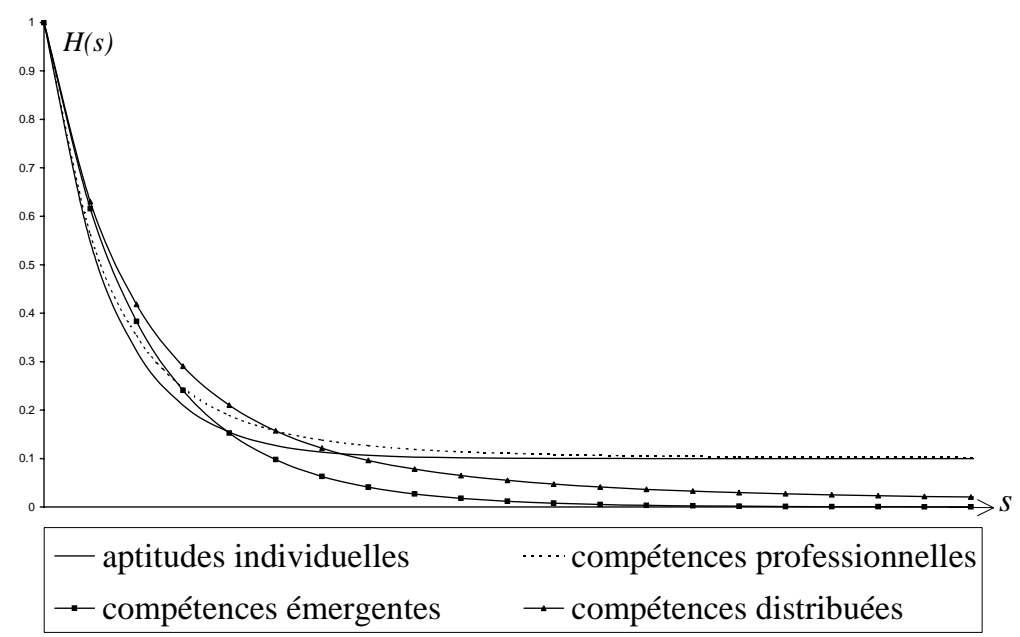

in F. Eymard-Duvernay (ed.), Valeurs, coordination et rationalité - développements, La Découverte, coll. Recherche, avril, pp. 111-127 
GRAPHIQUE 4. LES FONCTIONS DE SURVIE SIMULÉES

DES QUATRE CONVENTIONS DE QUALITÉ AVEC FORTE SÉLECTION :

$$
\overline{\boldsymbol{a}}>0.5(\overline{\boldsymbol{a}}=0.7 ; \mu=0.7 ; \mathrm{V}=10)
$$

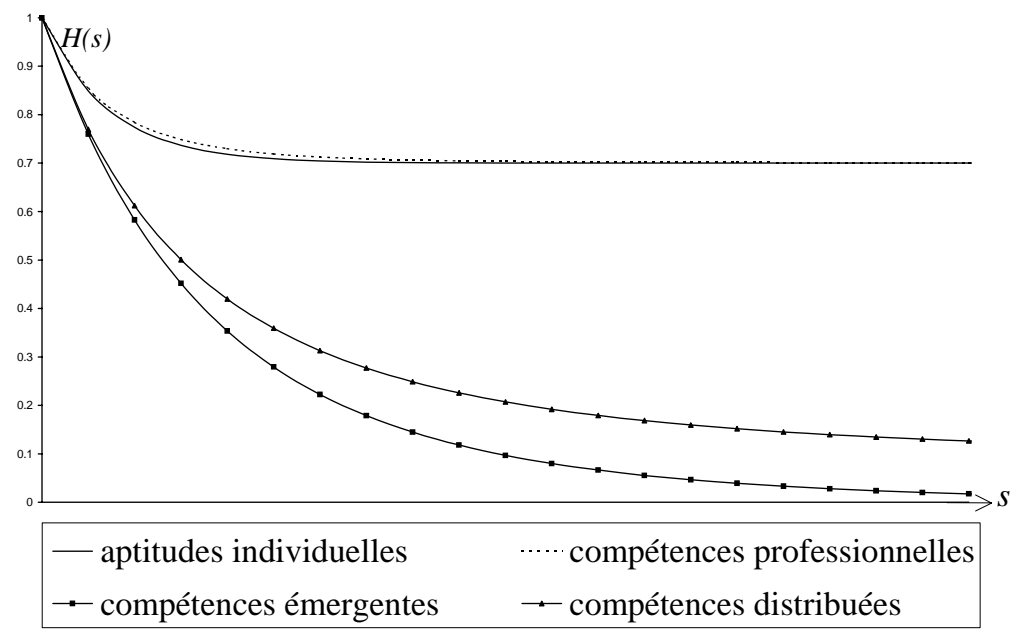

Quant aux graphiques 3 et 4, ils indiquent, pour une cohorte de chômeurs donnée, l'évolution avec la durée de chômage du pourcentage de chômeurs encore au chômage après une certaine période s. Ils donnent une idée de l'efficacité des modèles d'évaluation étudiés. Le degré de sélection a ici un impact non négligeable : plus il est fort, plus la proportion d'individus encore chômeurs après une certaine période est grande, quelle que soit la convention de qualité adoptée. Mais, à degré de sélection donné, ce sont les conventions «aptitudes individuelles » et « compétences professionnelles » les moins efficaces sur la durée, privant une plus grande proportion de chômeurs des chances de retour à l'emploi. Enfin, à mesure que la sélection du recrutement se durcit, le rendant plus inégalitaire, la convention 
« compétences émergentes » prend un avantage relatif en termes d'efficacité par rapport aux autres. Ce dernier s'explique par le renouvellement de l'évaluation, lui-même dû au caractère local du jugement, permettant au chômeur de ne pas être qualifié une fois pour toutes mais au contraire différemment lors de chaque évaluation.

Le paramètre de renouvellement joue donc un rôle important en termes d'équité et d'efficacité. La négociation des compétences de manière locale peut en effet empêcher les exclusions durables. Rappelons cependant que le paramètre de renouvellement est conditionné par celui de valeur : selon la valeur sur laquelle s'appuie le jugement, le renouvellement de l'épreuve est rendu possible ou non. Et même si la convention « compétences émergentes » est celle qui permet le renouvellement le plus fréquent, tous les travailleurs ne sont pas «bons » lors d'un entretien où l'intuition et la subjectivité de l'évaluateur engage la décision. Ainsi, se fixer sur un unique paramètre de valeur pour juger les candidats à l'emploi (adopter une unique convention de qualité) correspond à un jugement « déséquilibré » [cf. EymardDuvernay et Marchal, 1997, p. 49], et de fait irréversible. Une manière d'obtenir un renouvellement fréquent de l'évaluation consiste à évaluer selon les quatre conventions pour que les qualifications attachées à chacune se compensent. Cela produit un jugement équilibré entre les quatre paramètres de valeurs considérés. L’employabilité des candidats n’est pas définitivement 
figée mais comprend un caractère local et émergent.

Concernant le paramètre d'inégalités, la comparaison des graphiques 3 et 4 reconnaît une plus grande efficacité à une évaluation moins sélective. Un jugement équilibré sur une base peu sélective apparaît tout aussi équitable qu'efficace. À l'inverse, un jugement fondé sur une seule convention, de surcroît inégalitaire, correspond à une conception de l'employabilité universelle et à son corollaire, l’inemployabilité définitive, ce qui crée davantage d'exclusion. Le risque est pourtant grand d'adopter un tel jugement déséquilibré qui respecte au plus serré la contrainte économique : il est moins coûteux d'écarter certains candidats à partir du seul tri de CV sans affiner l'évaluation par des tests psychotechniques, un entretien et la mobilisation d'un réseau. Si tout recrutement commence par un tri de $\mathrm{CV}$, ce sont toujours les mêmes individus qui seront exclus d'une possible évaluation, à l'inverse d'un jugement équilibré qui permet d'éviter les exclusions durables.

\section{Conclusion}

Les recruteurs ne sont pas les seuls à mobiliser les conventions de qualité du travail pour évaluer les chômeurs, d'autres intermédiaires du marché du travail, notamment le personnel des agences de placement, les mobilisent quand ils appliquent les mesures de politiques publiques. Typiquement, le profilage est 
une méthode statistique d'évaluation, innovée par la politique de l'emploi américaine, qui attribue à tout chômeur une note chiffrée, calculée sur la base d'informations multivariées. Cet outil administratif aide les intermédiaires du marché du travail à une orientation plus efficace des demandeurs d'emploi vers les services de réemploi [OCDE, 2002, Eberts, 1999, Herbillon, 2004]. Le profilage repose sur la pertinence des modèles statistiques à relier la probabilité de réemploi à des caractéristiques personnelles et aux conditions du marché du travail local. Il donne un cadre aux déterminants supposés de l'employabilité puisqu'il repère les demandeurs d'emploi jugés les moins employables, pour une prise en charge rapide.

Cette méthode statistique est généralisée aux États-Unis, en Australie, aux Pays-Bas, et a été expérimentée au Royaume-Uni. Les informations multivariées utilisées dans ces pays pour attribuer une note d'employabilité aux chômeurs concernent principalement les compétences professionnelles (niveau d'instruction, ancienneté, dernière profession) et/ou les aptitudes individuelles ( "maîtrise de la langue », "assurance dans la recherche d'emploi », " capacité à valoriser ses propres compétences »), correspondant finalement aux valeurs des conventions pour lesquelles les « qualités » sont universelles (selon notre typologie), c'est-à-dire les seules conventions donnant un sens à la notion de population employable.

Il existe des méthodes dont la logique prend le contre-pied des mesures comme le profilage, focalisées sur le chômeur et ses 
caractéristiques. Se développe par exemple en France la méthode IOD (Intervention sur l'Offre et la Demande) ${ }^{10}$. Cette méthode considère d'une part que la notion d'employabilité correspond à un construit social, i.e. relève d'une convention, qui dépend du contexte, aussi bien sur le plan théorique (produit de croyances et de représentations) que sur le plan pratique (l'employabilité se construit et se révèle en situation réelle de travail). Elle suppose d'autre part que l'exclusion des personnes fragilisées ne résulte pas d'un comportement rationnel de la part des employeurs mais de pratiques de «sur-sélection » fondées sur des stéréotypes sociaux. La conception locale et émergente de l'employabilité rapproche la méthode de la convention «compétences émergentes ». La méthode s'appuie sur une évaluation locale en provoquant des rencontres directes avec l'employeur; elle induit également une modification des dispositifs de recrutement puisque les équipes travaillent sans $\mathrm{CV}$ ni lettre de motivation. L'intermédiaire va ainsi pouvoir focaliser l'attention de l'employeur-évaluateur sur d'autres points que les critères des modèles d'évaluation les plus répandus.

10 Cf. l'étude de la méthode IOD de Salognon [2004].

in F. Eymard-Duvernay (ed.), Valeurs, coordination et rationalité - développements, La Découverte, coll. Recherche, avril, pp. 111-127 


\section{Références Bibliographiques}

BenARRosh Y. (2000), « Tri des chômeurs : le nécessaire consensus des acteurs de l'emploi. », Travail et Emploi, n 81, p. 9-26.

Bessy C., Eymard-Duvernay F., de Larquier G., Marchal E. (2001), Des marchés du travail équitables? Une approche comparative France Royaume-Uni, Peter Lang - Presses Interuniversitaires Européennes, Bruxelles.

Decreuse B., Di Paola V. (2002), «L'employabilité des chômeurs de longue durée - mise en perspective des littératures théorique et empirique », Revue d'Économie Politique, n² 2, p. 197-227.

EBerts R.W. (1999), «The Use of Profiling in the United States for Early Identification and Referral of Less Employable Unemployment Insurance Recipients », in GAzIER B. (Ed.), Employability, Concepts and Policies, , Institute for Applied Socio-economics, Berlin, p. 121-144.

Eymard-Duvernay F. (2001), «Principes de justice, chômage et exclusion : approfondissements théoriques ", in BEssy et alii (Eds.), Des marchés du travail équitables? Une approche comparative France Royaume-Uni, , p. 271-297.

Eymard-Duvernay F., MARChal E. (1997), Façons de recruter. Le jugement des compétences sur le marché du travail, Métailié, Paris.

FAVEREAU O. (1986), «La formalisation du rôle des conventions dans l'allocation des ressources ", in SALAIS R., THÉvENot L. (Eds.), Le travail: marché, règles, conventions, Économica, Paris, p. 249268. 
Herbillon J.-M. (2004), «Le 'profiling' des demandeurs d'emploi aux Pays-Bas », Travail et Emploi, n 99, p. 7-25.

KIEFER N. (1988), « Economic Duration Data and Hazard Functions », Journal of Economic Literature, n² 2, p. 646-679.

MARChAL E., TORNY D. (2003), «Des petites aux grandes annonces : le marché des offres d'emploi depuis 1960 », Travail et Emploi, $\mathrm{n}^{\circ}$ 95, p. 59-71.

OCDE (2002), « Le chômage de longue durée : tenants et aboutissants », Perspectives de l'emploi de l'OCDE, Paris, p. 205-260.

SALOGNON M. (2004), « Modes de jugement des entreprises et insertion des chômeurs de longue durée : observation de la méthode IOD », Colloque Matisse «L'accès inégal à l'emploi et à la protection sociale », 16-17 septembre.

SiMONIN B. (2000), « Rechercher un emploi quand on est au chômage », Premières Informations et Premières Synthèses, nº 16.1. 